\title{
Efeito do jateamento de partículas na resistência de união e comportamento mecânico de cerâmicas à base de zircônia - Revisão
}

\section{(Effect of particle sandblasting on the bond strength and mechanical behavior of zirconia-based ceramics - Review)}

\author{
J. C. Mosele, M. Borba \\ Faculdade de Odontologia da Universidade de Passo Fundo, BR286, Bairro S. José, Passo Fundo, RS, \\ Brasil 99052-900
}

\begin{abstract}
Resumo
As cerâmicas ácido-resistentes como a zircônia tetragonal parcialmente estabilizada com ítria (Y-TZP), podem ter melhorada sua resistência de união ao cimento resinoso por meio de abrasão por jateamento de partículas. Entretanto, não existe uma padronização dos parâmetros utilizados nesse jateamento, o que pode afetar negativamente o comportamento mecânico da Y-TZP. O objetivo deste estudo foi buscar, através de uma revisão da literatura, quais são os protocolos de jateamento utilizados e qual protocolo resulta em melhores valores de resistência de união ao cimento resinoso e menor degradação das propriedades mecânicas da Y-TZP. A estratégia de pesquisa envolveu uma busca de artigos na base de dados online Medline/Pubmed, de 2003 a 2013, utilizando as palavras chaves: zircônia, adesão, jateamento e resistência mecânica (zirconia, bond strength, sandblasting, and mechanical strength). Foram selecionados vinte e cinco artigos, que estavam de acordo com os critérios de inclusão. O tamanho de partícula mais utilizado foi de $50 \mu \mathrm{m}$ para partículas de óxido de alumínio e de 110 $\mu \mathrm{m}$ para as partículas de óxido de alumínio modificadas por sílica. Segundo os estudos, o tamanho de partícula e o tempo de jateamento não influenciam os valores de resistência de união. Entretanto, o maior tamanho e o tipo de partícula (óxido de alumínio) afetam negativamente a resistência mecânica da Y-TZP. Ainda existem controvérsias a respeito do efeito da pressão na resistência de união e na resistência mecânica da Y-TZP. O uso de pressões muito baixas pode afetar os valores de adesão. A distância entre a ponta jateadora e a superfície cerâmica e o tempo variou entre 7 a $30 \mathrm{~mm}$ e 5 a $90 \mathrm{~s}$, respectivamente. Pôde-se concluir que alguns parâmetros do protocolo de jateamento ainda não estão bem definidos. Mesmo assim, os resultados dos estudos parecem corroborar a recomendação do uso de partículas pequenas e pressões baixas para alcançar o melhor resultado de adesão sem comprometer a resistência mecânica do material.
\end{abstract}

Palavras-chave: cerâmica; abrasão dental por ar.

\begin{abstract}
The bond strength of acid-resistant ceramics, such as yttria partially stabilized tetragonal zirconia (Y-TZP), to resin cements could be improved by surface sandblasting with particles. However, there is not a standard protocol for sandblasting, which may compromise Y-TZP mechanical behavior. The objective of this study was to search, trough a literature review, which sandblasting protocols have been used and which one results in higher bond strength values of Y-TZP to resin cement and less degradation of the mechanical properties of Y-TZP. The research involved articles published from 2003 to 2013 using Medline/Pubmed online database and the keywords: zirconia, bond strength, sandblasting, and mechanical strength. Twenty five articles that were within the study inclusion criteria were selected. The particle size most frequently used was 50 um for aluminum oxide and $30 \mu \mathrm{m}$ for silica-modified aluminum oxide. According to the literature, the particle size and sandblasting time have no influence on the bond strength values. On the other hand, the larger particle size and the particle type (aluminum oxide) negatively affected the Y-TZP mechanical strength. There are disagreements regarding the effects of sandblasting pressure on the bond and Y-TZP mechanical strength. The use of extremely low pressures may decrease the bond strength values. The distance from the tip of the sandblasting device and the ceramic surface, and the sandblasting time, varied from 7 to $30 \mathrm{~mm}$ and 5 to $90 \mathrm{~s}$, respectively. It can be concluded that some of the sandblasting parameters are not yet clearly defined. Even though, the results of studies seem to confirm the recommendation of using small particles and low sandblasting pressures to achieve superior bond strength without decreasing the material mechanical strength.
\end{abstract}

Keywords: ceramics, air abrasion, dental. 


\section{INTRODUÇÃO}

A crescente utilização de cerâmicas na fabricação de restaurações dentárias deve-se principalmente a três fatores: estética, biocompatibilidade e boas propriedades mecânicas [1-4]. A cerâmica à base de zircônia tetragonal parcialmente estabilizada com ítria (Y-TZP) tem alcançado grande popularidade na Odondologia devido a seus altos valores de resistência à fratura (900-1000 MPa) e tenacidade à fratura $\left(5,5-7,4 \mathrm{MPa} \cdot \mathrm{m}^{1 / 2}\right)$, associados ao mecanismo de tenacificação por transformação de fase (transformation toughness) [4]. As restaurações à base de Y-TZP são produzidas utilizando o sistema CAD/CAM (Computer Aided Design/Computer Aided Manufacturing - Desenho e manufatura auxiliados por computador) e, devido a sua alta opacidade, a Y-TZP é utilizada como material de infraestrutura, sendo recoberta com uma cerâmica vítrea com melhores propriedades ópticas para obter uma restauração com cor e translucidez semelhante à estrutura dental [5].

A composição e a microestrutura das cerâmicas e as propriedades químicas e físicas dos agentes de cimentação influenciam significativamente na natureza do mecanismo de união e durabilidade das restaurações de cerâmicas. Tratamentos de superfície são comumente utilizados com o objetivo de aumentar a área de superfície interna das restaurações, criando uma superfície áspera e limpa (isenta de impurezas) com energia favorável para união entre a cerâmica e o substrato dental [6]. Assim, para a cimentação adesiva, a microestrutura das cerâmicas pode ser ácido-sensível ou ácido-resistente [6,7], de acordo com a capacidade de reação química com primers específicos (ex: ácido hidrofluorídrico) [8].

Cerâmicas com alto conteúdo (fração) amorfo são mais sensíveis ao condicionamento ácido, já que o ácido dissolve preferencialmente a fase vítrea e/ou a interface entre a fase cristalina e a vítrea. A superfície das cerâmicas condicionadas mostra diferentes padrões de condicionamento dependendo do tipo de primer, concentração e tempo utilizado [9]. Já é conhecido que para as cerâmicas ácido-sensíveis, a associação entre o condicionamento com ácido hidrofluorídrico (HF) e a aplicação de um agente de união silano tem promovido excelentes valores de resistência de união e durabilidade adesiva (união mecânica e química) $[6,10]$. Por outro lado, as cerâmicas ácido-resistentes, em essência composta por uma microestrutura policristalina monofásica, como a alumina e a Y-TZP, podem ter sua resistência de união melhorada por meio de abrasão por jateamento de partículas de óxido de alumínio, modificadas ou não por sílica. O jateamento com partículas de alumina é capaz de produzir micro-rugosidades para o embricamento micromecânico com o cimento. Ainda, por meio da abrasão de partículas modificadas por sílica (ex. Rocatec e Cojet) é possível depositar uma camada de sílica na superfície das cerâmicas (efeito triboquímico). O resultado é uma superfície coberta com uma fina camada de sílica, que além de proporcionar uma potencial retenção micromecânica também promove sítios para adesão química por meio da aplicação de agentes de união como o silano [11]. O tratamento químico com cimentos adesivos ou primers específicos a base de monômeros bifuncionais (ex: MDP) também é indicado [12-14].

A técnica de jateamento com partículas de óxido de alumínio é fácil e simples de ser executada. Por isso, essa técnica é amplamente utilizada na Odontologia para a cimentação adesiva de restaurações confeccionadas com cerâmicas ácido-resistentes. No jateamento, as partículas são movidas em um fluxo de ar e se chocam contra a superfície interna das restaurações cerâmicas. O tamanho de partícula de alumina, dureza, velocidade, morfologia, fluxo e ângulo de impacto determinam a proporção da abrasão e, consequentemente, o padrão de rugosidade criado na superfície cerâmica [15]. Entretanto, não existe uma padronização dos parâmetros utilizados no jateamento de partícula. Assim, o principal desafio da aplicação clínica deste tratamento de superfície é encontrar um protocolo capaz de produzir um padrão de microrugosidade superficial que melhore a união com o cimento resinoso sem produzir defeitos que possam comprometer a resistência à fratura da restauração cerâmica. Portanto, o objetivo deste estudo foi buscar, através de uma revisão da literatura, quais são os protocolos de jateamento utilizados nas pesquisas e qual protocolo resulta em melhores valores de resistência de união do cimento resinoso à cerâmica Y-TZP sem comprometer sua resistência mecânica.

\section{MÉTODOS}

A estratégia de pesquisa envolveu uma busca a partir da base de dados online Medline/Pubmed, utilizando a seguinte combinação de palavras chaves: zircônia, adesão, jateamento e resistência mecânica (zirconia, bond strength, sandblasting, mechanical strength). Foram pesquisados artigos publicados de 2003 a 2013. Foram incluídos na revisão de literatura artigos in vitro que investigaram exclusivamente a influência de diferentes parâmetros do jateamento de partículas: na resistência de união da Y-TZP ao cimento resinoso e na resistência mecânica da Y-TZP.

\section{RESULTADOS}

Foram selecionados vinte e cinco artigos, que estavam de acordo com os critérios de inclusão da revisão de literatura. Os parâmetros de jateamento e principais resultados dos artigos estão na Tabela I e II. Nos estudos, as principais variações nos protocolos de jateamento foram: tamanho de partícula $(30,110$ e $125 \mu \mathrm{m}$ para partícula de óxido de alumínio modificada por sílica; e 25, 45, 50, 70, $75,100,110,125$ e $150 \mu \mathrm{m}$ para partícula de óxido de alumínio, sem modificação); pressão de jateamento $(0,05$ a 0,60 MPa); tempo de jateamento (5 a $90 \mathrm{~s})$ e distância entre a superfície cerâmica e o jato $(10 \mathrm{a} 30 \mathrm{~mm})$. 
Tabela I - Resultados encontrados nos estudos de resistência de união entre a Y-TZP e o cimento resinoso para a influência do jateamento de partículas, discriminando o tipo (TiP) e tamanho (TaP) de partícula, a pressão (P), distância (D) e o tempo (T) utilizados no protocolo de jateamento.

[Table I - Literature findings for the influence of sandblasting on the bond strength of Y-TZP to resin cement, describing the particle's type (TiP) and size (TaP), the pressure (P), distance (D) and time (T) used in the sandblasting protocol.]

\begin{tabular}{|c|c|c|c|c|c|c|}
\hline Estudo & TiP & $\begin{array}{c}\mathrm{TaP} \\
(\mu \mathrm{m})\end{array}$ & $\begin{array}{c}\mathrm{P} \\
(\mathrm{MPa})\end{array}$ & $\begin{array}{c}\mathrm{D} \\
(\mathrm{mm})\end{array}$ & $\begin{array}{l}\mathrm{T} \\
(\mathrm{s})\end{array}$ & Resultados \\
\hline $\begin{array}{l}\text { Tsuo et al. } \\
\text { (2006) }\end{array}$ & OA & $\begin{array}{c}50 \\
75 \\
100 \\
150\end{array}$ & 0,38 & 10 & 20 & $\begin{array}{l}\text { O jateamento com partículas maiores produziu maiores } \\
\text { valores de rugosidade. Os valores de resistência de união } \\
\text { foram estatisticamente iguais para todos os grupos testados. }\end{array}$ \\
\hline $\begin{array}{l}\text { Yang et al. } \\
\text { (2007) }\end{array}$ & OA & 50 & 0,25 & 10 & 15 & $\begin{array}{c}\text { O jateamento foi o único instrumento eficaz na limpeza da } \\
\text { superfície da cerâmica após a contaminação. }\end{array}$ \\
\hline $\begin{array}{l}\text { Heikkinen } \\
\text { et al. } \\
(2007)\end{array}$ & OAS & 30 & $\begin{array}{l}0,15 \\
0,22 \\
0,30 \\
0,45\end{array}$ & 10 & 10 & $\begin{array}{l}\text { A resistência de união aumentou com o aumento da pressão } \\
\qquad(0,15 \text { para } 0,45 \mathrm{MPa}) .\end{array}$ \\
\hline $\begin{array}{l}\text { Re et al. } \\
(2008)\end{array}$ & OAS & $\begin{array}{c}50 \\
110 \\
110\end{array}$ & 0,25 & 10 & 10 & $\begin{array}{c}\text { O jateamento aumentou os valores de resistência de união. } \\
\text { O jateamento com OAS obteve os maiores valores para a } \\
\text { cerâmica do tipo Lava (3M ESPE). Para a cerâmica Cercon } \\
\text { (Dentsply) a resistência de união foi semelhante quando } \\
\text { usado OA ou OAS. }\end{array}$ \\
\hline
\end{tabular}

\begin{tabular}{cccccccc}
\hline $\begin{array}{c}\text { Kern et al. } \\
(2009)\end{array}$ & OA & 50 & 0,05 & $*$ & $*$ & $\begin{array}{c}\text { As duas pressões investigadas melhoraram de forma igual a } \\
\text { resistência de união, juntamente com monômeros fosfatados. }\end{array}$ \\
\hline $\begin{array}{c}\text { Attia e Kern } \\
(2011)\end{array}$ & OA & 50 & 0,25 & 10 & 15 & $\begin{array}{c}\text { O jateamento foi eficaz na durabilidade de união } \\
\text { para ambas as pressões, onde o método de limpeza } \\
\text { com ultrassom pode ser recomendado. }\end{array}$ \\
\hline $\begin{array}{c}\text { Castro et al. } \\
(2012)\end{array}$ & OA & $\leq 45$ & 0,19 & $*$ & 20 & & $\begin{array}{c}\text { A resistência de união foi semelhante entre os grupos jateados } \\
\text { com OA e OAS testados após 3 dias e termociclados, mas foi } \\
\text { estatisticamente diferente para grupo armazenado em água } \\
\text { por } 6 \text { meses, onde o grupo jateado com OAS alcançou um } \\
\text { resultado melhor. }\end{array}$ \\
\hline
\end{tabular}

OA $\quad 50 \quad 0,05 \quad 10 \quad 20$

Komine 0,10

et al. $\quad 0,20$

A resistência de união foi estatisticamente igual para as

pressões de $0,1,0,2,0,4$ e $0,6 \mathrm{MPa}$.

(2012)

0,40

\begin{tabular}{cccccccc}
\hline & & 0,60 & & & \\
$\begin{array}{c}\text { Re et al. } \\
(2012)\end{array}$ & OA & 50 & 0,10 & 10 & 20 & \\
& & 0,25 & & & $\begin{array}{c}\text { O jateamento aumentou a resistência de união adesiva, onde a } \\
\text { pressão maior foi estatisticamente diferente da pressão menor. }\end{array}$ \\
\hline $\begin{array}{c}\text { Shimoe et al. } \\
(2012)\end{array}$ & OA & $50-70$ & 0,30 & 10 & 10 & A variação do tempo não alterou a resistência de união. \\
\hline
\end{tabular}

Notas: TiP: OA = óxido de alumínio; OAS = óxido de alumínio modificado por sílica; D - distância entre a superfície do corpo-de-prova e a ponta do aparato de jateamento; * - informação não fornecida pelo estudo.

Notes: TiP: $O A=$ aluminum oxide; $O A S=$ silica-modified aluminum oxide; $D$ - distance from the tip of the sandblasting device to the specimen surface;

* - information not reported in the study. 
Tabela II - Resultados encontrados nos estudos para a influência do jateamento de partículas na resistência mecânica da Y-TZP, discriminando o tipo (TiP) e tamanho (TaP) de partícula, a pressão (P), distância (D) e o tempo (T) utilizados no protocolo de jateamento.

[Table II - Literature findings for the influence of sandblasting on the mechanical strength of Y-TZP, describing the particle's type $(\mathrm{TiP})$ and size $(\mathrm{TaP})$, the pressure $(P)$, distance $(D)$ and time $(T)$ used in the sandblasting protocol.]

\begin{tabular}{|c|c|c|c|c|c|c|}
\hline Estudo & TiP & $\begin{array}{c}\mathrm{TaP} \\
(\mu \mathrm{m})\end{array}$ & $\begin{array}{c}\mathrm{P} \\
(\mathrm{MPa})\end{array}$ & $\mathrm{D}$ & & Resultados \\
\hline $\begin{array}{l}\text { Zhang et al. } \\
(2004)\end{array}$ & $\mathrm{OA}$ & 50 & 0,28 & 10 & 5 & $\begin{array}{l}\text { O jateamento com partículas de óxido de alumínio } \\
\text { reduziu a resistência à fadiga em } 30 \% \text {. }\end{array}$ \\
\hline $\begin{array}{l}\text { Guazzato et al. } \\
\quad(2005)\end{array}$ & $\mathrm{OA}$ & 110 & 0,5 & $*$ & 20 & $\begin{array}{l}\text { As amostras jateadas produziram os maiores valores de } \\
\text { resistência à flexão em três pontos e módulo de Weibull. }\end{array}$ \\
\hline $\begin{array}{l}\text { Curtis et al. } \\
\quad(2006)\end{array}$ & $\mathrm{OA}$ & $\begin{array}{l}25 \\
50 \\
110\end{array}$ & 0,48 & 20 & 5 & $\begin{array}{l}\text { O jateamento com diferentes tamanhos de partícula aumentou a resistência à } \\
\text { flexão biaxial e o módulo de Weibull da Y-TZP quando comparado com } \\
\text { o grupo controle (sem jateamento), tanto em ambiente seco como úmido. }\end{array}$ \\
\hline $\begin{array}{l}\text { Zhang et al. } \\
\text { (2006) }\end{array}$ & $\mathrm{OA}$ & 50 & 0,28 & 10 & 5 & $\begin{array}{l}\text { A resistência à fadiga de cerâmicas à base de zircônia } \\
\text { diminuiu após o jateamento. }\end{array}$ \\
\hline $\begin{array}{l}\text { Kosmac et al. } \\
\quad(2007 \mathrm{a})\end{array}$ & $\mathrm{OA}$ & 110 & 0,4 & 30 & 15 & $\begin{array}{c}\text { O jateamento não afetou a resistência à flexão biaxial após a ciclagem } \\
\text { mecânica das amostras, que apresentaram o módulo de Weibull } \\
\text { maior que o grupo controle (sem jateamento). }\end{array}$ \\
\hline $\begin{array}{l}\text { Kosmac et al. } \\
\quad(2007 b)\end{array}$ & $\mathrm{OA}$ & 110 & 0,4 & 30 & 15 & $\begin{array}{l}\text { O jateamento não afetou a resistência à flexão biaxial após a ciclagem } \\
\text { mecânica. As origens das fraturas não tiveram correlação com o jateamento. }\end{array}$ \\
\hline $\begin{array}{l}\text { Sato et al. } \\
(2008 \mathrm{a})\end{array}$ & $\begin{array}{c}\text { OA } \\
\text { OAS }\end{array}$ & $\begin{array}{c}70 \\
125\end{array}$ & 0,40 & 10 & 10 & $\begin{array}{l}\text { O jateamento com partículas maiores produziu um aumento na rugosidade } \\
\text { e na quantidade de transformação de fase. Esta transformação de fase foi } \\
\text { considerada excessiva e negativa para a resistência à flexão biaxial. }\end{array}$ \\
\hline $\begin{array}{l}\text { Sato et al. } \\
(2008 b)\end{array}$ & $\begin{array}{l}\text { OA } \\
\text { OAS }\end{array}$ & $\begin{array}{c}70 \\
125\end{array}$ & 0,40 & 10 & $\begin{array}{l}10 \\
90\end{array}$ & $\begin{array}{l}\text { O jateamento com partículas de } 70 \mu \mathrm{m} \text { e } 125 \mu \mathrm{m} \text { produziram valores de } \\
\text { resistência à flexão biaxial semelhantes. Foi observado o aumento de } \\
\text { rugosidade e quantidade de transformação de fase para o grupo } \\
\text { jateado com partícula de } 125 \mu \mathrm{m} \text {, independente do tempo de jateamento. }\end{array}$ \\
\hline $\begin{array}{l}\text { Wang et al. } \\
\text { (2008) }\end{array}$ & $\mathrm{OA}$ & $\begin{array}{c}50 \\
120 \\
150\end{array}$ & 0,35 & 20 & 25 & $\begin{array}{l}\text { O jateamento com particulas de } 50 \mu \mathrm{m} \text { resultou em valores de resistência } \\
\text { à flexão em três pontos iguais ao do grupo controle (sem jateamento). Foi } \\
\text { observado que os processos de fabricação por meio da usinagem podem } \\
\text { comprometer a resistência mecânica do material cerâmico. }\end{array}$ \\
\hline $\begin{array}{l}\text { Aboushelib } \\
\text { e Wang } \\
(2010)\end{array}$ & OAS & 110 & 0,20 & 10 & $*$ & $\begin{array}{c}\text { A resistência à flexão em quatro pontos das amostras jateadas reduziu em média } \\
50 \% \text { quando comparada ao grupo controle (sem jateamento). } \\
\text { No entanto, para o grupo jateado e que recebeu uma camada de agente } \\
\text { adesivo, a resistência reduziu apenas } 19,2 \% \text {. }\end{array}$ \\
\hline $\begin{array}{l}\text { Aboushelib } \\
\text { (2010) }\end{array}$ & $\begin{array}{c}\mathrm{OA} \\
\mathrm{OAS}\end{array}$ & $\begin{array}{c}50 \\
110\end{array}$ & $\begin{array}{c}0,15 \\
030\end{array}$ & $*$ & $*$ & $\begin{array}{l}\text { A resistência à flexão em quatro pontos após a ciclagem mecânica só foi } \\
\text { estatisticamente igual para as amostras jateadas com partículas de } 50 \mu \mathrm{m} \text {. }\end{array}$ \\
\hline $\begin{array}{l}\text { Guess et al. } \\
\quad(2010)\end{array}$ & $\mathrm{OA}$ & 50 & 0,5 & 10 & 5 & $\begin{array}{l}\text { O comportamento mecânico das cerâmicas jateadas com partículas de } \\
\text { óxido de alumínio foi semelhante ao comportamento das cerâmicas } \\
\text { abrasionadas por discos. A confiabilidade estimada para o uso clínico de } 10^{5} \\
\text { ciclos com uma carga de } 200 \mathrm{~N} \text { foi de } 98 \% \text { para o grupo controle } \\
\text { (sem tratamento) e } 56 \% \text { para o grupo jateado. }\end{array}$ \\
\hline $\begin{array}{l}\text { Doi et al. } \\
(2011)\end{array}$ & $\mathrm{OA}$ & 50 & 0,30 & 15 & 15 & $\begin{array}{l}\text { A resistência à flexão em três pontos para o grupo jateado foi } \\
\text { estatisticamete igual ao grupo controle (sem jateamento). }\end{array}$ \\
\hline $\begin{array}{l}\text { Scherrer et al. } \\
\quad(2011)\end{array}$ & OAS & 30 & 0,25 & 7 & 20 & $\begin{array}{l}\text { O jateamento aumentou entre } 15 \% \text { a } 30 \% \text { a resistência } \\
\text { à flexão em quatro pontos após a ciclagem mecânica em } \\
\text { relação ao grupo controle (sem jateamento). }\end{array}$ \\
\hline $\begin{array}{l}\text { Iijima et al. } \\
(2013)\end{array}$ & $\mathrm{OA}$ & $\begin{array}{c}50 \\
150\end{array}$ & 0,40 & 10 & & $\begin{array}{l}\text { Os valores de resistência à flexão biaxial com o jateamento com partículas de } 50 \mu \mathrm{m} \\
\text { foram semelhantes ao grupo controle (sem jateamento). Esses valores decresceram } \\
46,1 \% \text { para o grupo jateado e } 37,4 \% \text { para o grupo controle após a ciclagem mecânica. }\end{array}$ \\
\hline
\end{tabular}




\section{DISCUSSÃO}

\section{Resistência de união}

Pôde-se observar que o jateamento com diferentes tamanhos de partícula e pressões afetam diretamente a topografia de superfície da cerâmica Y-TZP [16-20]. Foi avaliado o efeito de diferentes tamanhos de partícula de óxido de alumínio $(50,75,100,150 \mu \mathrm{m})$ na rugosidade de superfície da cerâmica Y-TZP e concluiu-se que o aumento da rugosidade é proporcional ao aumento do tamanho de partícula [20]. Este efeito também foi visualizado com a variação da pressão e o tempo de jateamento $[16,21]$. Por outro lado, não existe um consenso na literatura a respeito do aumento da resistência de união com o aumento da rugosidade proporcionada por diferentes tamanhos de partículas, pressões e tempos de jateamento. Os valores de resistência de união encontrados nos estudos diferem bastante por causa das diferenças metodológicas. $\mathrm{O}$ ensaio mais encontrado nos estudos foi o teste de cisalhamento seguido pelo teste de tração e microtração. Ainda, existem diferenças nos tipos de tratamento do substrato, ligante, aderente, tempo, tipo e intensidade de fotopolimerização dos componentes. Heintze [24], ao correlacionar os valores de resistência de união encontrados nos testes laboratoriais e a qualidade marginal observada in vivo, concluiu que não existem ainda um ensaio e parâmetros adequados para a análise da qualidade de união, já que muitos fatores estão envolvidos, tornando difícil a comparação dos resultados obtidos em diferentes estudos. Ainda assim, é importante fazer uma análise dos estudos para descobrir como esses diferentes parâmetros podem influenciar o comportamento de adesão da Y-TZP. A pressão foi um dos parâmetros que mais variou entre os estudos, sendo a pressão de $0,25 \mathrm{MPa}$ a mais utilizada. Foi investigada a influência da pressão de jateamento na resistência ao cisalhamento de dois tipos de cerâmica à base de Y-TZP e um cimento resinoso. A variação da pressão do jateamento $(0,15$ para $0,45 \mathrm{MPa})$ melhorou a resistência de união para ambos tipos de cerâmica Y-TZP. Entretanto, não existiram diferenças estatísticas na resistência de união quando a variação da pressão foi de 0,15 , 0,22 e $0,30 \mathrm{MPa}$, para a cerâmica Lava, e entre as pressões 0,15 e $0,22 \mathrm{MPa}$ e 0,30 e $0,45 \mathrm{MPa}$ para a cerâmica Procera Zirconia [25].

Uma investigação que também avaliou o efeito da pressão de jateamento na resistência de união da Y-TZP ao cimento resinoso relatou que o jateamento com a pressão de 0,05 $\mathrm{MPa}$ proporcionou o menor valor de resistência de união. As pressões de 0,10, 0,20,0,40 e 0,60 MPa resultaram em valores de resistência de união semelhantes [17]. Outro estudo também observou diferentes valores de resistência de união quando a pressão testada variou de 0,10 para $0,25 \mathrm{MPa}$, utilizando partículas de óxido de alumínio de $50 \mu \mathrm{m}$ [19]. Por outro lado, Kern et al. [16] não observaram influência da pressão de jateamento na resistência à tração entre a cerâmica Y-TZP e o cimento resinoso. O tratamento de superfície dos corpos-de-prova foi realizado utilizando partículas de óxido de alumínio de $50 \mu \mathrm{m}$ e pressão de $0,05 \mathrm{MPa}$ ou $0,25 \mathrm{MPa}$.
Sem jateamento, os primers fosfatados utilizados melhoraram apenas a resistência inicial das cerâmicas. Não houve diferença estatística nos valores de resistência de união entre as duas pressões testadas, após armazenagem por 150 dias combinada com termociclagem. Este achado está de acordo com outra investigação, onde também não foi encontrada diferença estatística nos valores de resistência de união entre as pressões de $0,05 \mathrm{MPa}$ e $0,25 \mathrm{MPa}$ [21].

Outro parâmetro que variou nos estudos foi o tipo e tamanho de partícula utilizada no jateamento. A utilização de partículas de alumina modificadas por sílica teve como objetivo alcançar união micro-mecânica e química entre os substratos. A presença de sílica na superfície seria capaz de estabelecer uma união química com o agente silano. Entretanto, estudos que compararam a resistência adesiva da Y-TZP ao cimento resinoso utilizando o jateamento de partículas modificadas e o jateamento convencional encontraram valores de resistência semelhantes, mesmo quando tamanhos de partículas diferentes foram utilizados $[18,23]$. Diferenças foram observadas apenas após 6 meses de armazenagem, sendo que o grupo jateado com partículas modificadas obteve os melhores valores de resistência adesiva [23]. Considerando que os profissionais são mais familiarizados e têm um acesso mais fácil ao jateamento de partículas de alumina, esse protocolo tem sido amplamente utilizado para o tratamento de superfície de cerâmicas ácidoresistentes. Além disso, o jateamento com óxido de aluminío também se mostrou eficaz para a limpeza da superfície cerâmica contaminada com saliva e outros resíduos [26].

O tamanho de partícula mais utilizado nos estudos foi de $50 \mu \mathrm{m}$ para partículas de óxido de alumínio e de $30 \mu \mathrm{m}$ para as partículas de óxido de alumínio modificadas por sílica. A influência da variação do tamanho de partícula de óxido de alumínio na resistência de união da Y-TZP ao cimento resinoso foi avaliada, utilizando partículas de 50, 75,100 e $150 \mu \mathrm{m}$ com a pressão de $0,38 \mathrm{MPa}$. Concluiuse que o tamanho de partícula não teve efeito nos valores de resistência de união [20]. Resultado semelhante foi encontrado em outro estudo que, ao verificar a influência da variação do tamanho de partícula de óxido de alumínio (50 e $110 \mu \mathrm{m})$, não encontrou diferença estatística entre os grupos testados para a resistência de união [18]. A agressão mecânica provocada pelo jateamento com partículas de alumina também pode afetar a estabilidade da estrutura cristalina tetragonal da zircônia. Um estudo investigou, além do tempo de jateamento, a influência do tratamento térmico $\left(500{ }^{\circ} \mathrm{C}\right.$ com taxa de aquecimento de $50{ }^{\circ} \mathrm{C} / \mathrm{min}$ até $1200{ }^{\circ} \mathrm{C}$, onde permaneceu por $10 \mathrm{~min}$ ) após jateamento na resistência de união da Y-TZP ao cimento resinoso e na quantidade de fase monoclínica. Observaram que o conteúdo de zircônia monoclínica aumentou por causa do jateamento, e diminuiu após o tratamento térmico. Ainda, o tempo maior de jateamento de partículas de óxido de alumínio (10 s para 20 s) produziu maiores alterações na superfície, obtendo maiores valores de rugosidade média. Apesar disso, os valores de resistência de união foram similares para os dois tempos de jateamento testados [21]. 
O envelhecimento artificial é uma importante ferramenta para avaliar a estabilidade de união em condições de umidade e variações de temperatura, simulando o ambiente oral. Entre os trabalhos reportados na literatura, foram observadas variações no tempo de armazenamento dos corpos-de-prova antes do teste ( 1 dia a 6 meses, em água destilada a $37^{\circ} \mathrm{C}$ ) e, quando a termociclagem foi utilizada, também houve variação para o número de ciclos $(5.000 \mathrm{a}$ 37.000) e temperatura $\left(4{ }^{\circ} \mathrm{C}-60^{\circ} \mathrm{C}\right.$ ou $\left.5{ }^{\circ} \mathrm{C}-55^{\circ} \mathrm{C}\right)$. Apesar das significativas variações nas condições de testes, as falhas de união relatadas nos estudos após a termociclagem foram em sua maioria do tipo coesiva para os grupos que utilizaram em seus protocolos de cimentação o jateamento e monômeros bifuncionais fosfatados, sugerindo uma boa qualidade de união para esses protocolos [16, 17, 20, 21].

\section{Resistência mecânica da cerâmica Y-TZP}

A agressão mecânica provocada pelo jateamento com partículas introduz defeitos na superfície da Y-TZP, mas a natureza e caráter desses defeitos não são totalmente compreendidos. O aumento da rugosidade observados nos estudos é decorrente da perda superficial do material cerâmico devido aos impactos repetitivos das partículas. As partículas são movidas em um fluxo de ar e se chocam contra a superfície interna da restauração cerâmica gerando tensões de compressão e ocasionando uma deformação plástica na superfície afetada. Dependendo do impacto das partículas, tensões de tração são geradas em volta das áreas de deformação plástica gerando microfissuras paralelas e/ ou perpendiculares à superfície. Desta forma, para que a resistência mecânica não seja afetada, espera-se que os defeitos criados possam ser estabilizados por tensões de compressão em torno da trinca decorrentes da expansão volumétrica associada à transformação de fase da zircônia tetragonal (t) para monoclínica (m). Por isso, diferentes ensaios mecânicos têm sido propostos na literatura com o objetivo de verificar a correlação do tamanho e severidade dos defeitos criados pelo jateamento com a proporção de transformação de fase $(\mathrm{t}-\mathrm{m}) \mathrm{e}$ as propriedades mecânicas das cerâmicas de Y-TZP, procurando simular um carregamento de força que pode ser contínuo (estático) e/ou repetido (cíclico), a fim de acumular danos na microestrutura e induzir a falha [27-41].

A resistência à flexão em três pontos da Y-TZP foi avaliada após o jateamento de partículas de alumina, após a simulação dos efeitos de usinagem e após o tratamento térmico. O jateamento foi realizado com partículas de óxido de alumínio de $110 \mu \mathrm{m}$ e pressão de $0,5 \mathrm{MPa}$. A quantidade de transformação de fase $(\mathrm{t}-\mathrm{m})$ observada por difração de raios $\mathrm{X}$ (XRD) foi maior para o grupo jateado em comparação aos submetidos aos efeitos da usinagem e tratamento térmico. Foi observado que o tratamento térmico reverteu a transformação de fase induzida pelo jateamento, ou seja, observou-se que o conteúdo (fração) de zircônia monoclínica diminuiu significativamente após o ciclo térmico. Os autores verificaram que o jateamento promoveu uma superfície rugosa e severamente danificada, onde a dimensão dos defeitos críticos que deram origem à fratura ficaram em torno $6 \mu \mathrm{m}$. No entanto, os valores de resistência à flexão em três pontos como também o módulo de Weibull foram maiores para o grupo jateado. Os autores concluíram que o aumento da resistência à flexão após o jateamento está associado à maior quantidade de transformação de fase (t-m) [28]. Esses achados estão de acordo com outros estudos que também verificaram a influência do aumento da quantidade de fase monoclínica no aumento da resistência à flexão quando usado o jateamento, e a redução da resistência flexural quando promovida a reversão da fase $\mathrm{m}$-t após o tratamento térmico. Ainda, foi observado que a transformação da fase monoclínica não é controlada pelo tempo de jateamento, mas sim pelo tamanho de partículas, onde partículas maiores $(70$ para $125 \mu \mathrm{m})$ promoveram uma transformação de fase monoclínica excessiva. Segundo os autores, essa transformação deve ser controlada para que não tenha um efeito negativo sobre a resistência flexural ou na longevidade da restauração cerâmica [33,34]. Outra investigação também observou que o jateamento com partícula de tamanho $120 \mu \mathrm{m}$ diminuiu a resistência flexural e que partículas menores (50 $\mu \mathrm{m})$ não afetaram a resistência flexural quando comparado com o grupo controle polido. No entanto, não foi observada redução da resistência flexural após o tratamento térmico [34, 38]. Ainda, Wang [35], ao analisar a superfície cerâmica, observou que ambos os tamanhos de partícula produziram defeitos de superfície, onde o maior tamanho de partícula produziu defeitos que estendiam até $30 \mu \mathrm{m}$. Ao testar as amostras que simularam o processo de fabricação por meio da usinagem verificou-se que a resistência flexural do material foi menor do que das amostras polidas. Os autores ressaltam que os processos de fabricação introduzem defeitos capazes de comprometer a resistência mecânica dos materiais cerâmicos e que a escolha de qualquer instrumento que possa introduzir mais defeitos deve ser visto com cautela para a longevidade das restaurações de zircônia. Ainda, a relação dos processos de fabricação com a introdução de defeitos mais severos do que os induzidos pelo jateamento é relatado por outras investigações $[29,31,32,40]$.

Para avaliar in vitro a longevidade dos materiais cerâmicos e observar a influência do jateamento de partículas, ensaios de fadiga cíclica tem sido proposto, fazendo com que os defeitos estáveis inicialmente cresçam de forma lenta ocasionando, a longo prazo, a falha do sistema cerâmico (subcritical crack gowth - SCG). Iijima et al. [41] avaliaram a resistência à flexão biaxial de cerâmicas Y-TZP submetidas aos processos jateamento com alumina e condicionadas com ácido hidrofluorídrico a $47 \%$. Os autores verificaram que o aumento da rugosidade foi diretamente dependente do tratamento proposto, sendo maior para os grupos jateados com partícula de tamanho de $150 \mu \mathrm{m}$. Foi relatado que a resistência à flexão biaxial estática foi estatisticamente igual ao grupo controle (sem jateamento) somente quando utilizado o jateamento com partículas de $50 \mu \mathrm{m}$. Ainda, a resistência à flexão biaxial após a ciclagem $\left(10^{6}\right.$ ciclos $)$ da Y-TZP decresceu em 37,4\% para o grupo controle, e para os 
grupos jateados com partícula de tamanho de $50 \mu \mathrm{m}$ e 150 $\mu \mathrm{m}$ decresceu $46,1 \%$ e $44,0 \%$, respectivamente. A quantidade de fase monoclínica foi igual antes e após a ciclagem. Os grupos jateados com o maior tamanho de partícula obtiveram um maior aumento na quantidade de fase monoclínica. Desta forma, os autores concluíram que os defeitos ocasionados pelo jateamento poderiam ser responsáveis pelo decréscimo na resistência mecânica [41], mas não relataram se os valores de resistência à flexão após a ciclagem das amostras jateadas com partícula de $50 \mu \mathrm{m}$ foram estatisticamente diferentes ao grupo controle. Outra investigação verificou que os resultados de resistência à flexão de quatro pontos após a ciclagem (250.000 ciclos) foram estatisticamente semelhantes ao grupo controle (sem jateamento) somente quando utilizadas partículas de $50 \mu \mathrm{m}$ [36]. Nesse sentido, Scherrer et al. [40] relataram o aumento entre 15 a $30 \%$ da resistência à flexão em quatros pontos após a ciclagem $\left(10^{6}\right.$ ciclos) de diferentes cerâmicas de Y-TZP jateadas com óxido de alumínio modificado por sílica (tamanho $30 \mu \mathrm{m}$; pressão 0,25 MPa). Sendo que a análise fractográfica revelou que as falhas estavam relacionadas aos processos de usinagem (direção de corte dos blocos) e a presença de defeitos da fabricação dos materiais, mas não ao processo de jateamento. Além disso, os autores observaram que após o jateamento houve suavização dos defeitos produzidos pelo processo de usinagem, que são possíveis concentradores de tensão.

O efeito dos sistemas adesivos após o jateamento também foi investigado. $\mathrm{O}$ adesivo seria capaz de selar e modificar as falhas de superfície proporcionada pelo jateamento [37]. No entanto, Guess et al. [38] avaliaram o comportamento da interface adesiva (restauração, cimento e substrato) quando as cerâmicas à base de Y-TZP foram submetidas ao jateamento por partícula de óxido de alumínio e ciclagem mecânica e concluíram que as cerâmicas jateadas apresentaram um comportamento mecânico diferente do grupo controle (sem jateamento). Ao simular a aplicação de uma carga de 200 $\mathrm{N}$ por 100.000 ciclos, a confiabilidade obtida por meio da análise de Weibull foi de $56 \%$ para o grupo jateado e $98 \%$ para o grupo controle. Outros estudos também verificaram a redução da resistência mecânica da cerâmica Y-TZP quando jateadas por partículas óxido de alumínio [27, 30]. Ainda, sugere-se que as partículas de óxido de alumínio se caracterizam por ser duras e com cantos agudos, e que o uso de abrasivos mais suaves como as partículas de óxido de alumínio modificadas por sílica (partículas arredondadas e macias) deve ser explorado nas investigações [30].

Assim, não existe um consenso na literatura a respeito dos danos microestruturais ocasionados por diferentes tamanhos de partículas, pressões e tempos de jateamento e sua influência na resistência mecânica da cerâmica Y-TZP o que torna preocupante extrapolar os achados nos resultados dos ensaios in vitro para a situação clínica.

\section{CONSIDERAÇÕES FINAIS}

Os parâmetros de jateamento, como tamanho de partículas, pressão e tempo, para maximização tanto da resistência de união do cimento resinoso à cerâmica Y-TZP como da resistência mecânica da cerâmica Y-TZP jateada ainda não estão bem definidos. $\mathrm{O}$ tamanho de partícula de óxido de alumínio $(45-150 \mu \mathrm{m})$ e o tempo de jateamento (10 a 20 s) não têm influência na resistência de união entre a Y-TZP e o cimento resinoso. O uso de partículas de óxido de alumínio de maior tamanho $(110 \mathrm{a} 150 \mu \mathrm{m})$ pode diminuir a resistência mecânica da Y-TZP. O tamanho de partícula mais utilizado nos estudos foi de $50 \mu \mathrm{m}$ para partículas de óxido de alumínio e de $110 \mu \mathrm{m}$ para as partículas de óxido de alumínio modificadas por sílica. Existem controvérsias a respeito do efeito da pressão de jateamento, tanto para a resistência de união quanto para a resistência mecânica da Y-TZP. A recomendação do uso de partículas pequenas e pressões baixas como melhor opção de protocolo de jateamento de partículas para a Y-TZP está em acordo com os resultados reportados em literatura.

\section{REFERÊNCIAS}

[1] S. Spear, J. Holloway, "Which all-ceramic system is optimal for anterior esthetics?", J. Am. Dent. Assoc. 139, (2008) 19S-24S.

[2] D. Edelhoff, J. A. Sorensen, "Tooth structure removal associated with various preparation designs for posterior teeth", Int. J. Periodontics. Restorative. Dent. 22 (2002) 241-9.

[3] D. Edelhoff, J. A. Sorensen, "Tooth structure removal associated with various preparation designs for anterior teeth", J. Prosthetic. Dent. 87 (2002) 503-9.

[4] M. Guazzato, M. Albakry, S. P. Ringer, M. V. Swain, "Strength, fracture toughness and microstructure of a selection of all-ceramic materials. Part II. Zirconia-based dental ceramics", Dent. Mater. 20 (2004) 449-56.

[5] J. R. Strub, E. D. Rekow, S. Witkowski, "Computeraided desing and fabrication of dental restorations: current systems and future possibilities", J. Am. Dent. Assoc. 137 (2006) 1289-96.

[6] A. Della Bona, "Characterizing ceramics and the interfacial adhesion to resin: II - The relationship of surface treatment, bond strength, interfacial toughness and factography”, J. Appl. Oral. Sci. 13 (2005) 101-9.

[7] M. A. Vargas, C. Bergeron, A. Diaz-arnold, "Cementing all-ceramic restorations Recommendations for success", J. Am. Dent. Assoc. 142 (2011) 20S-4S.

[8] G. A. Borges, A. M. Sophr, M. F. De Goes, L. C. Sobrinho, D. C. Chan, "Effect of etching and airborne particle abrasion on the microstructure of different dental ceramics", J. Prosthet. Dent. 89, 5 (2003) 479-88.

[9] A. Della Bona, K. J. Anusavice, "Microstructure, composition, and etching topography of dental ceramics", Int. J. Prosthodont. 15 (2002) 159-67.

[10] P. Pospiech, "All-ceramic crowns: bonding or cementing?”, Clin. Oral. Invest. 6 (2002) 189-197.

[11] A. Della Bona, M. Borba, P. Benetti, D. Cecchetti, "Effect of surface treatments on the bond strength of zirconia-reinforced ceramic to composite resin”, Braz. Oral 
Res. 21 (2007) 10-5.

[12] M. Kern, S. M. Wegner, "Bonding to zirconia ceramic: adhesion methods and their durability", Dent. Mater. 14 (1998) 64-71.

[13] M. B. Blatz, G. Chiche, S. Holst, A. Sadan, “ Influence of surface treatment and simulated aging on bond strengths of luting agents to zirconia”, Quintessence. Int. 38 (2007) 745-753.

[14] J. P. Matinlinna, T. Heikkinen, M. Özcan, L. V. Lassila, P. K. Vallittu, "Evaluation of resin adhesion to zirconia ceramic using some organosilanes", Dent. Mater. 22 (2006) 824-831.

[15] O. Addison, P. M. Marquis, G. J. Fleming, "The impact of modifying alumina air abrasion parameters on the fracture strength of a porcelain laminate restorative material", Dent. Mater. 23 (2007) 1332-41.

[16] M. Kern, A. Barloi, B. Yang, "Surface conditioning influences zirconia ceramic bonding", J. Dent. Res. 88 (2009) 817-22.

[17] F. Komine, R. Fushiki, M. Koizuka, K. Taguchi, S. Kamio, H. Matsumura, "Effect of surface treatment on bond strength between an indirect composite material and a zirconia framework", J. Oral. Sci. 54 (2012) 39-46.

[18] D. Re, D. Augusti, I. Sailer, D. Spreafico, A. Cerutti, "The effect of surface treatment on the adhesion of resin cements to Y-TZP”, Eur. J. Esthet. Den. 3 (2008) 186-96.

[19] R. Re, D. Augusti, G. Augusti, A. Giovannetti, "Early bond strength to low-pressure sandblasted zirconia: evaluation of a self-adhesive cement", Eur. J. Esthet. Dent. 7 (2012) 164-75.

[20] Y. Tsuo, K. Yoshida, M. Atsuta, "Effects of aluminablasting and adhesive primers on bonding between resin luting agent and zirconia ceramics", Dent. Mater. J. 25 (2006) 669-74.

[21] S. Shimoe, N. Tanoue, K. Kusano, M. Okazaki, T. Satoda, "Influences of air-abrasion and subsequent heat treatment on bonding between zirconia framework material and indirect composites", Dent. Mater. J. 31, (2012) 751-7.

[22] A. Attia, N. Kern, "Effect of cleaning methods after reduced-pressure air abrasion on bonding to zirconia ceramic", J. Adhes. Dent. 13 (2011) 561-7.

[23] H. L. de Castro, P. H. Corazza, T. de A. Paes-Júnior, A. Della Bona, "Influence of Y-TZP ceramic treatment and different resin cements on bond strength to dentin", Dent. Mater. 28 (2012) 1191-7.

[24] S. D. Heintze, "Systematic reviews: I. The correlation between laboratory tests on marginal quality and bond strength. II. The correlation between marginal quality and clinical outcome", J. Adhes. Dent. 1 (2007) 77-106.

[25] T. T. Heikkinen, L. V. Lassila, J. P. Matinlinna, P. K. Vallittu, "Effect of operating air pressure on tribochemical silica-coating", Acta. Odontol. Scand. 65 (2007) 241-8.

[26] B. Yang, S. Wolfart, M. Scharnberg, K. Ludwing, R. Adelung, M. Kern, "Influences of contamination on zirconia ceramics bonding", J. Dent. Res. 86 (2007) 749-53.

[27] Y. Zhang, B. R. Lawn, E. D. Rekow, V. P. Thompson,
"Effect of sandblasting on the long-term performance of dental ceramics", J. Biomed. Mater. Res. B. Appl. Biomater. 15 (2004) 381-6.

[28] M. Guazzato, L. Quach, M. Albakry, M. V. Swain, "Influence of surface and heat treatments on the flexural strength of Y-TZP dental ceramic", J. Dent. 33, 1 (2005) 9-18.

[29] A. R. Curtis, A. J. Wright, G. J. Fleming, "The influence of surface modification techniques on the performance of a Y-TZP dental ceramic", J. Dent. 34 (2006) 195-206.

[30] Y. Zhang, B. R. Lawn, Malament K. A. V. Thompson, E. D. Rekow, "Damage accumulation and fatigue life of particle-abraded ceramics", Int. J. Prosthodont. 19 (2006) 442-8.

[31] T. Kosmac, A. Dakskobler, C. Oblak, P. Jevnikar, "The strength and hydrothermal stability of Y-TZP ceramics for dental applications", Int. J. Ceram. Technol. 4 (2007) 16474.

[32] T. Kosmac, C. Oblak, P. Jevnikar, "The fracture and fatigue of surface-treated tetragonal zirconia (Y-TZP) dental ceramics", Mater. Technol. 41 (2007) 237-41.

[33] H. Sato, K. Yamanda, G. Pezzotti, M. Nawa, S. Ban, "Mechanical properties of dental zirconia ceramics changed with sandblasting and heat treatment", Dent. Mater. 27 (2008) 408-14.

[34] H Sato, D. Yamashita, S. Ban, "Relation between biaxial flexure and phase transformation of zirconia with surface treatments", J. Ceram. Soc. Jpn. 116 (2008) 28-30.

[35] H. Wang, M. N. Aboushelib, A. J. Feilzer, "Strength influencing variables on CAD/CAM zirconia frameworks", Dent. Mater. 24 (2008) 633-8.

[36] M. N. Aboushelib, "Long term fatigue behavior of zirconia based dental ceramics", Mater. 3 (2010) 2975-85.

[37] M. N. Aboushelib, H. Wang, "Effect of surface treatment on flexural strength of zirconia bars", J. Prosthet. Dent. 104 (2010) 98-104.

[38] P. C. Guess, Y. Zhang, J. W. Kim, E. D. Rekow, V. P. Thompson, "Damage and reliability of Y-TZP after cementation surface treatment", J. Dent. Res. 89 (2010) 592-6.

[39] M. Doi, K. Yoshida, M. Atsuta, T. Sawase, "Influence of pre-treatments on flexural strength of zirconia and debonding crack-initiation strength of veneered zirconia", J. Adhes. Dent. 13 (2011) 79-84.

[40] S. S. Scherrer, M. Cattani-lorente, E. Vittecop, F. De Mestral, J. A. Griggs, H. W. Wiskott, "Fatigue behavior in water of Y-TZP zirconia ceramics after abrasion with $30 \mu \mathrm{m}$ silica-coated alumina particles”, Dent. Mater. 27 (2011) 2842.

[41] T. Iijima, S. Homma, H. Sekine, H. Sasaki, Y. Yajima, M. Yoshinaro, "Influence of surface treatment of yttriastabilized tetragonal zirconia polycrystal with hot isostatic pressing on cyclic fatigue strength", Dent. Mater. J. 32 (2012) 274-80.

(Rec. 23/05/2013, Rev. 19/08/2013, 17/10/2013, Ac. $15 / 11 / 2013)$ 